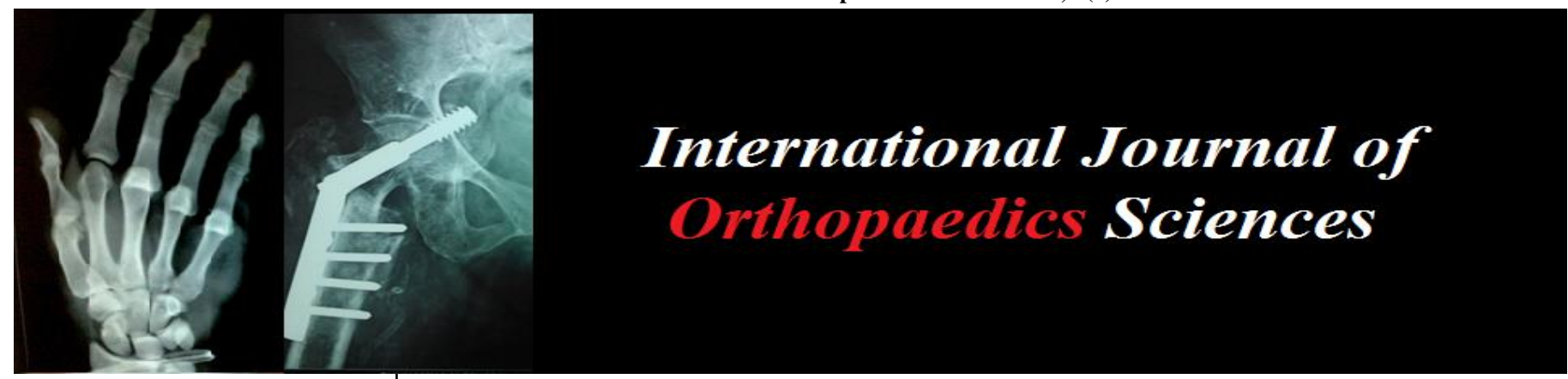

E-ISSN: 2395-1958

P-ISSN: 2706-6630

IJOS 2020; 6(2): 900-903

(C) 2020 IJOS

www.orthopaper.com

Received: 04-02-2020

Accepted: 05-03-2020

Dr. Rajesh K Ambulgekar Professor and Head, Department of Orthopaedics, Dr Shankarrao Chavan Govt Medical College,

Nanded, Maharashtra, India

Dr. Dhananjay E More Junior Resident, Department of Orthopaedics, Dr Shankarrao

Chavan Govt Medical College,

Nanded, Maharashtra, India

Dr. Akshay Kharat

Junior Resident, Department of Orthopaedics, Dr Shankarrao

Chavan Govt Medical College,

Nanded, Maharashtra, India
Corresponding Author: Dr. Dhananjay E More Junior Resident, Department of Orthopaedics, Dr Shankarrao Chavan Govt Medical College, Nanded, Maharashtra, India

\section{A case report of: Aneurysmal bone cyst in proximal tibia treated with simple curettage with bone graft}

\author{
Dr. Rajesh K Ambulgekar, Dr. Dhananjay E More and Dr. Akshay Kharat
}

DOI: https://doi.org/10.22271/ortho.2020.v6.i2o.2157

\section{Abstract}

Aneurysmal bone cyst is a benign bone tumor occurring in the metaphysis of long bones of immature skeleton commonly. We present a case of aneurysmal bone cyst of proximal tibial metaphysis treated by curettage and bone grafting followed by one year followup. It was concluded that early detection and prompt treatment leads to reduced need of repeated surgeries.

Keywords: Proximal tibia, Aneurysmal bone cyst, bone graft

\section{Introduction}

Aneurysmal bone cyst are benign bone lesion. ABC is also called as multilocular or hematinic cyst. Diagnosis of ABC is made with various imaging modalities and biopsy. Primary ABC is typically seen to affect individuals in first two decades. The lesion is usually solitary however multiple site involvement in same patients has also been reported and can be seen in $5 \%$ of patients. ABCs cause pain and swelling in close proximity to affected bone. As ABCs commonly manifest in paediatric patients, growth plate involvement and permanent limb length deformities are of great concern. By far majority of aneurysmal bone cyst present in metaphysis of long bone (femur, tibia, humerus). Due to its aggressive bony erosion, $\mathrm{ABC}$ can lead to pathological fracture. In axial spine the sacrum, pelvis (represent $50 \%$ of flat bony lesions) and vertebrae have been reportedly involved. Within the spine, the lesion may cause neurological symptoms secondary to mass effect impinging on the spinal cord or exiting nerve roots. There are several approaches for treatment of ABC. Currently most widely employed treatment for $\mathrm{ABC}$ is intralesional curettage with or without bone graft.

Epidemiology: $\mathrm{ABC}$ are a rare osseous tumour comprising $1 \%$ to $6 \%$ of primary osseous tumours. The majority of patients diagnosed with an aneurysmal bone cyst are children and adolescents less than 20 years old.

Presentation of Case: A 12 year old female came to our hospital complaining of pain and swelling in her right leg. Initially she experienced pain in right leg and 3 months later she noticed a swelling in her right leg. On examination, there was a swelling in right leg without tenderness. The plain radiograph showed a multilocular, eccentric, expansile lesion in right proximal tibia with clear trabeculations and cortical thinning.

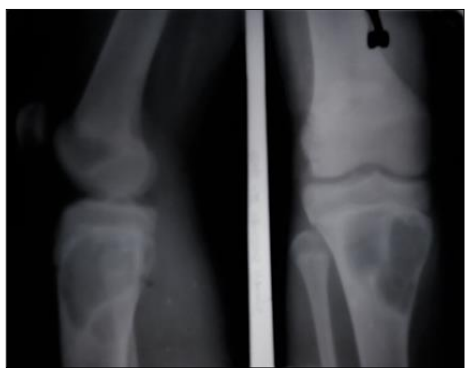

Fig 1: Plain radiograph of knee with leg showing a multilocular, eccentric lesion with cortical thinning $\sim 900 \sim$ 
MRI of right proximal tibia was suggestive of large expansile, multiloculated, lytic cystic lesion measuring $54 \mathrm{~mm}$ x $45 \mathrm{~mm}$ in size, involving metaphysis of tibia, mainly on medial condyle with fluid -fluid level, mild peri-focal and soft tissue edema, multiple cystic necrotic areas and periosteal reaction.
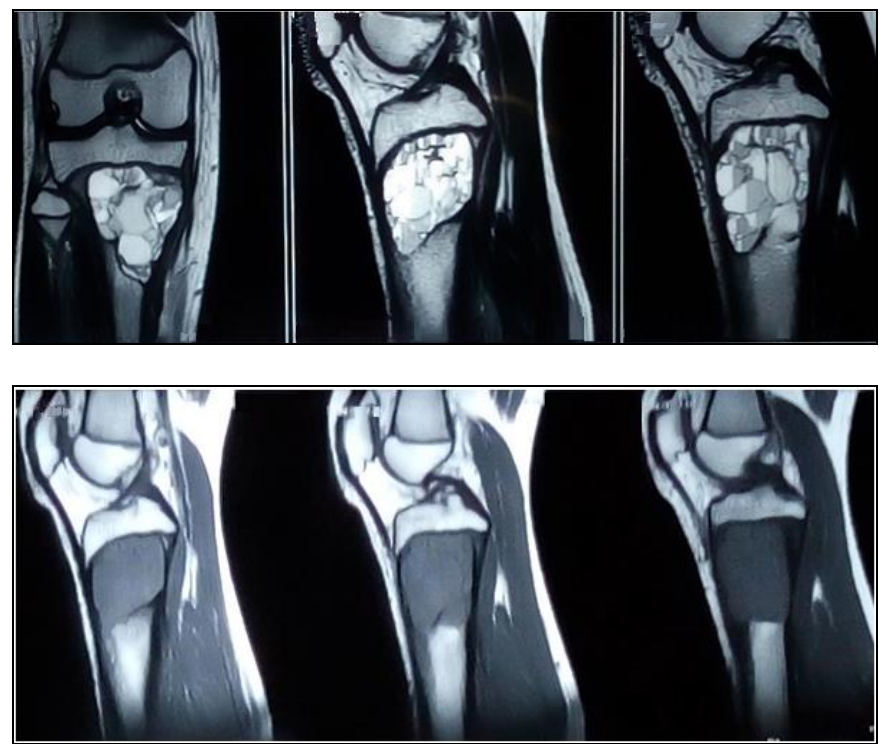

Fig 2 and Fig 3: MRI of proximal tibia showing multiloculated, lytic, cystic lesion involving metaphysis of medial condyle with fluid-fluid level and soft tissue edema

The lesion was extending upto epiphyseal plate with minimal edema in epiphysis. First fine needle aspiration and cytological examination was performed and the aspirated fluid gave the impression of reddish haemorrhagic fluid. Histopathological examination gave the impression of cyst wall with microscopic features of fibrocollagenous stroma, bony trabeculae and hemosiderin laden macrophages.
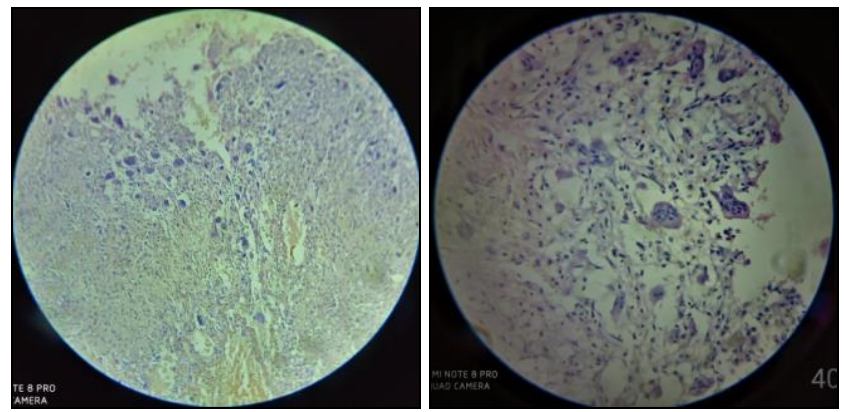

Fig 4: Histopathological evaluation of mass under low power and high power fields showing bony trabeculae and hemosiderin laden macrophages.

The lesion was surgically intervened with simple curettage and reconstruction of the defect with bone graft. Histological examination of the surgical specimen showed an aneurysmal bone cyst with fibro-collagenous stroma, bony trabeculae, medium to large sized spaces with multi-nucleated giant cells. An operative procedure was performed after which the pain was observed to decrease immediately. Radiographically the operated bone exhibited good remodelling and no recurrence of the lesion after the operation. After few months, she returned to all activities without any pain or limitation.
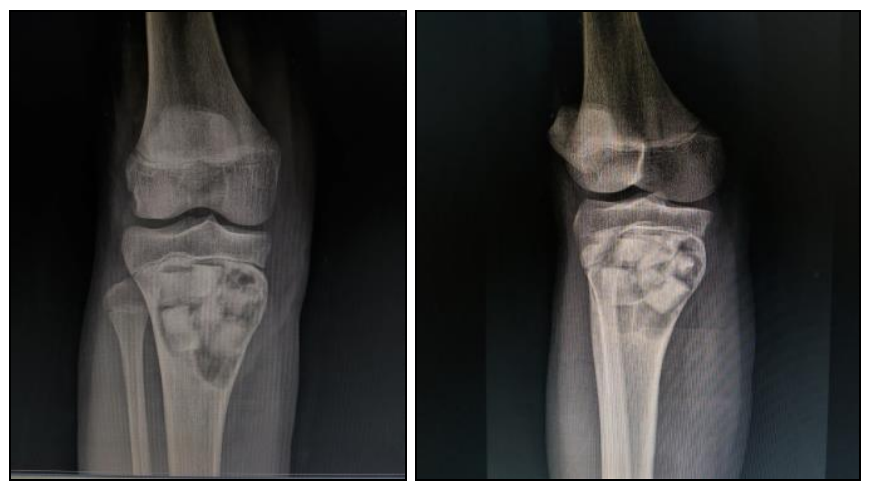

Fig 5: Anteroposterior and lateral radiographs of knee with leg at postoperative day 1

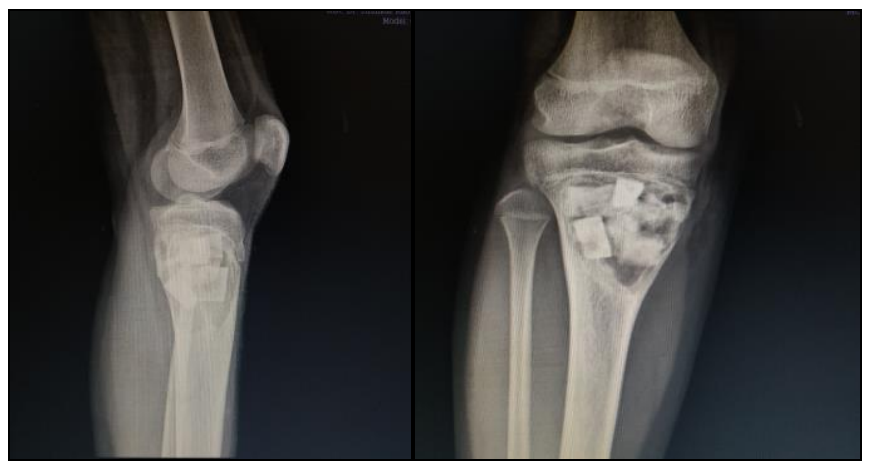

Fig 6: Anteroposterior and lateral radiographs of knee with leg at 3 months follow up showing uptake of artificial bone graft
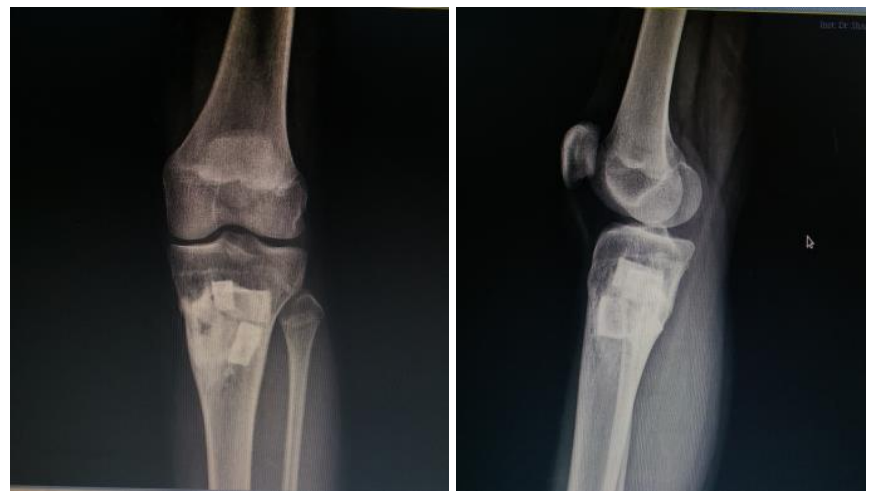

Fig 7: Anteroposterior and lateral radiographs of knee with leg at 12 months follow up showing remodelling of the proximal tibial bone and complete graft incorporation

\section{Discussion}

Osseous tumour containing giant cells are $\mathrm{ABC}$, GCT, Brown tumour, GCRG. Their differentiation is crucial. In GCT tumour is located mainly in epiphysis of bone which exhibit lytic expansion and eccentric location. GCRG originate from periosteal connective tissue having low mitotic activity. It tends to form cyst and exhibit reactive bone formation histologically characterised by a predominance of giant and mononuclear cells in areas of haemorrhage. Brown tumours have been reported in patient with chronic renal failure with lobulated architectural growth pattern wherein hyperthyroidism should be ruled out. The standard treatment for $A B C$ is curettage with or without bone graft. Due to the highly variable recurrence rate seen after curettage, several adjuvants have been used over past few years like cryotherapy, cement, phenol, high speed burr, argon beam coagulation. 
Cryotherapy includes the use of liquid or aerosolised nitrogen that creates freezing temperatures that leads to a cytotoxic effect on the ABC lesion .Cryotherapy is done following curettage of the lesion. Cryotherapy though has a low recurrence rate, is still not used widely as it has often led to complications like postoperative fracture, skin necrosis and wound infection ${ }^{[1,4]}$.

Polymethylmethacrylate (PMMA) cement is a biologically inert substance that helps in stabilisation of cavity in paediatric bone lesion and also as a recurrence reducing adjuvant through its exothermic reaction ${ }^{[5]}$. But PMMA also increases the risk of pathological fracture since it lacks the potential for osseous incorporation and has no growth potential. PMMA may also acts as a foreign body thereby acting as a nidus for infection.

Phenol is used to sterilise the lesion as it helps in removal of the residual neoplastic cells in the lesion ${ }^{[6]}$. Phenol with curettage has a low recurrence rate as compared to curettage alone ${ }^{[6]}$.

High speed burr augments the process of curettage by mechanical disruption of the lesion thereby helping to increase the cure rate. High speed burr is not reported to decrease the recurrence rates.

Argon beam coagulation is a process that creates a unipolar electric current through the tissue and produces dessication and coagulation which helps in reducing the recurrence rate of ABC when used following curettage [7]. Argon beam coagulation also runs the risk of developing a postoperative fracture by osteonecrosis and dessication ${ }^{[8]}$.

There also have been alternate treatment strategies in the form of adjuvant radiotherapy,arterial embolization and sclerotherapy in addition to standard procedure of curettage with use of adjuvants .

Selective arterial embolization is used when the lesion is difficult to assess like ABC located in pelvis, sacrum or the lesion at considerable risk of haemorrhage. It remains a limited treatment option since it results in skin necrosis, transient paresis and lesion may lack identifiable feeding vessels whichmakes it difficult to use SAE [9].

Sclerotherapy acts by destroying the endothelium of blood vessels resulting in the coagulation cascade, thrombosis and sclerosis of the vascular network resulting in local control of the lesion. Ethibloc is a radiopaque alcohol based sclerosant that causes local fibrinogenic and thrombogenic effect upon contact with $\mathrm{ABC}$ but results in aseptic bone necrosis ,pulmonary bone embolism, deep venous thrombosis and cerebellar infract which has lead to its reduction in utilisation $[10,14]$. Polidocanol used in treatment of varicose veins, is another sclerosant that also helps in providing faster pain relief, better functional outcomes, avoids the morbidity and costs of surgery $[11,15]$

Emerging techniques for the management of $\mathrm{ABC}$ include less aggressive surgical techniques and medical managements in the form of Curopsy, Bisphosphonates, Percutaneous Doxycyline, RANKL inhibition and Denosumab.

Bisphosphonates through their antineoplastic effects like induction of apoptosis, inhibition of tumour cell adhesion and invasion helps in pain relief in inoperable benign lesion like $\mathrm{ABC}{ }^{[16,17]}$.

Percutaneous doxycycline is a drug with antimicrobial and anti-neoplastic properties. It inhibits angiogenesis and matrix metalloproteinase, both of which helps in expansion bone cyst $[18,19]$.

Denosumab is a monoclonal antibody that inhibits the RANKL singling pathway. It is used as a neo adjuvant therapy for osteolytic bone lesions including ABC [20]. It also reduces the tumour size and decreases the morbidity of surgical interventions in $\mathrm{ABC}$.

\section{Conclusion}

Although most aneurysmal bone cyst are benign bone lesion rarely they may spread locally very aggressively. Recurrence is also common with these lesions. Early diagnosis and prompt adequate therapy can help to reduce events of recurrence and repeated surgeries.

\section{References}

1. Marcove RC, Sheth DS, Takemoto S, Healey JH. The treatment of aneurysmal bone cyst. Clin Orthop Relat Res. 1995; (311):157-63. Available at: http://www.ncbi.nlm.nih.gov/pubmed/7634571.

[PubMed]

2. Marcove RC, Weis LD, Vaghaiwalla MR, Pearson R, Huvos AG. Cryosurgery in the treatment of giant cell tumors of bone. A report of 52 consecutive cases. Cancer. 1978; 41(3):957-69. doi: 10.1002/10970142(197803)41:3<957::AID-

CNCR2820410325>3.0.CO;2-Y. [PubMed]

[CrossRef] [Google Scholar]

3. Schreuder HW, Veth RP, Pruszczynski M, Lemmens JA, Koops HS, Molenaar WM et al. Aneurysmal bone cysts treated by curettage, cryotherapy and bone grafting. J Bone Joint Surg (Br). 1997; 79(1):20-5. doi: 10.1302/0301-620X.79B1.7097. [PubMed]

[CrossRef] [Google Scholar]

4. Peeters SP, Van der Geest ICM, de Rooy JWJ, Veth RPH, Schreuder HWB. Aneurysmal bone cyst: the role of cryosurgery as local adjuvant treatment. J Surg Oncol. 2009; 100(8):719-24. doi: 10.1002/jso.21410. [PubMed] [CrossRef] [Google Scholar

5. Wallace MT, Henshaw RM. Results of cement versus bone graft reconstruction after intralesional curettage of bone tumors in the skeletally immature patient. J Pediatr Orthop. 2014; 34(1):92-100. doi: 10.1097/BPO.0b013e31829b2f61.

6. Capanna R, Sudanese A, Baldini N, Campanacci M. Phenol as an adjuvant in the control of local recurrence of benign neoplasms of bone treated by curettage. Ital J Orthop Traumatol. 1985; 11(3):381-

7. Lewis VO, Wei A, Mendoza T, Primus F, Peabody T, Simon MA et al. Argon beam coagulation as an adjuvant for local control of giant cell tumor. Clin Orthop Relat Res. 2007; 454:192-7. doi: 10.1097/01.blo.0000238784.98606.d4.

8. Steffner RJ, Liao C, Stacy G et al. Factors associated with recurrence of primary aneurysmal bone cysts: is argon beam coagulation an effective adjuvant treatment? J Bone Joint Surg Am. 2011; 93(21):e1221-9. doi: 10.2106/JBJS.J.01067.

9. Rossi G, Rimondi E, Bartalena T et al. Selective arterial embolization of 36 aneurysmal bone cysts of the skeleton with N-2-butyl cyanoacrylate. Skelet Radiol. 2010; 39(2):161-7. doi: 10.1007/s00256-009-0757-z.

10. Falappa P, Fassari FM, Fanelli A et al. Aneurysmal bone cysts: treatment with direct percutaneous Ethibloc injection: long-term results. Cardiovasc Intervent Radiol. 25(4):282-90. doi:10.1007/s00270-001-0062-2.

11. Varshney MK, Rastogi S, Khan SA, Trikha V. Is sclerotherapy better than intralesional excision for treating aneurysmal bone cysts? Clin Orthop Relat Res. 
2010; 468(6):1649-59. doi: 10.1007/s11999-009-1144-8.

12. Adamsbaum C, Mascard E, Guinebretière JM, Kalifa G, Dubousset J. Intralesional Ethibloc injections in primary aneurysmal bone cysts: an efficient and safe treatment. Skelet Radiol. 2003; 32(10):559-66. doi: 10.1007/s00256-003-0653-X.

13. Topouchian V, Mazda K, Hamze B, Laredo JD, Penneçot GF. Aneurysmal bone cysts in children: complications of fibrosing agent injection. Radiology. 2004; 232(2):522-6. doi: 10.1148/radiol.2322031157.

14. Peraud A, Drake JM, Armstrong D, Hedden D, Babyn P, Wilson $\mathrm{G}$ et al. Fatal ethibloc embolization of vertebrobasilar system following percutaneous injection into aneurysmal bone cyst of the second cervical vertebra. AJNR Am J Neuroradiol. 25(6):1116-20. Available at: http://www.ncbi.nlm.nih.gov/pubmed/15205161

15. Rastogi S, Varshney MK, Trikha V, Khan SA, Choudhury B, Safaya R et al. Treatment of aneurysmal bone cysts with percutaneous sclerotherapy using polidocanol. A review of 72 cases with long-term followup. J Bone Joint Surg (Br). 2006; 88(9):1212-6. doi: 10.1302/0301-620X.88B9.17829

16. Morgan G, Lipton A. Antitumor effects and anticancer applications of bisphosphonates. Semin Oncol. 2010; 37(2):S30-40. doi: 10.1053/j.seminoncol.2010.10.005.

17. Cornelis F, Truchetet ME, Amoretti $\mathrm{N}$ et al. Bisphosphonate therapy for unresectable symptomatic benign bone tumors: a long-term prospective study of tolerance and efficacy. Bone. 2014; 58:11-6. doi: 10.1016/j.bone.2013.10.004

18. Duivenvoorden WC, Hirte HW, Singh G. Use of tetracycline as an inhibitor of matrix metalloproteinase activity secreted by human bone-metastasizing cancer cells. Invasion Metastasis. 1997; 17(6):312-22. [PubMed] [Google Scholar]

19. Fife RS, Rougraff BT, Proctor C, Sledge GW. Inhibition of proliferation and induction of apoptosis by doxycycline in cultured human osteosarcoma cells. J Lab Clin Med. 1997; 130(5):530-4. doi: 10.1016/S00222143(97)90130-X.

20. Dubory A, Missenard G, Domont J, Court C. Interest of denosumab for the treatment of giant-cells tumors and aneurysmal bone cysts of the spine. About nine cases. Spine (Phila Pa 1976). 2016; 41(11):E654-60. doi: 10.1097/BRS.0000000000001350 